\title{
Chinese-Chess Image Recognition by using Feature Comparison Techniques
}

\author{
Wen-Yuan Chen* \\ Department of Electronic Engineering, National Chin-Yi University of Technology, Taichung, Taiwan, R.O.C.
}

Received: 12 Sep. 2013, Revised: 10 Dec. 2013, Accepted: 11 Dec. 2013

Published online: 1 Sep. 2014

\begin{abstract}
In this paper, we develop a feature comparison method for the Chinese-chess object by using the features comparison based on input image and database. Features are generated by calculating the distance between the contour of the character and the centre of the chess object. In this paper, the noise filter, object extraction, normalization, feature calculation (FC) and maximum energy slop (MES) method are used to achieve robust Chinese-chess recognition. There are two advantages when compared with other methods. 1) Our method is robust against the 40 incline degree attacks. 2) Our method can resist the $20 \%$ pepper and salt noise attacks. In order to demonstrate the effectiveness of the proposed scheme, simulations under all kinds of various conditions were conducted. The experimental results show that our proposed scheme can exactly identify chess images at $100 \%$ of accuracy under less than $20 \%$ noise added and 40 degree incline test environment condition.
\end{abstract}

Keywords: Chinese-chess, Image recognition, Object contour, Center of gravity, Feature comparison.

\section{Introduction}

Chinese-chess is a kind of traditional game for two people playing chess in Asia area. There are many playing methods varied by the playing rules such as alignments, playing peoples, and shapes of chess board. There are two situations to decide who the winner is; one is the winner who kills the king of the opposite side. The other is the loser who gives up the game.

In Chinese-chess research, peoples always focus on deploying the troops in battle formation, and do not pay attention to chess image identification. It is always focus on the intelligent playing game of human and neglect the playing with machine. In many chess playing machines $[1,2,3]$, they all exclude the chess image identification.

In C. Wang et al. [4] proposed an Adaptive Genetic Algorithm (AGA) to solve the problem of computer Chinese chess especial on the image identification. They describe the system on four parts: searching engine, move generator, evaluation function, and opening book. In their research, evaluation function associated with the parameters is designed. By the way, the combination of evaluation function is automatically adjusted and optimized by using tournament scheme and an independent self-study system designed.
Chen et al. [5] proposed a method of three sides'game searching with pruning. It is put forward based on a vector evaluation function. At the same time, they compared the three sides'Chinese game with the traditional Chinese chess. In the paper, they describe a machine learning scheme in detail by learning from history game processes. From the simulation results, it demonstrates the pruning and learning effect.

Fan et al. [6] proposed an improved Chinese chess computer programs to reduce the memory storage. They make each match that the movement of the opponent and the position value are stored in database, and avoid making the same mistake next time. The more it stores, the better it performs. Meanwhile, they also develop a new game tree search scheme. The experiment demonstrates the effectiveness and the feasibility of the proposed method.

Chen et al. [7] using a semi-automatic strategy to construct a large set of endgame heuristics, which is called endgame knowledge database. It is used in their program. Meanwhile, they also handle the problem of conflict based on large amount of knowledge. By applying the semi-automatic construction and modification process, they get a consistent endgame knowledge database.

\footnotetext{
*Corresponding author e-mail: cwy@ncut.edu.tw
} 
Barik and Mondal [8] presented a method for object identify on chaotic background by using image segmentation and graph partitioning. They build and train a "feature set" from the original object based on the chaotic image. From the computer and real world images simulation results, it is demonstrated that both cases on their system have identified the search object among other similar objects successfully.

Lifeng et al. [9] proposed a fast labeling method in binary image. By the way, their paper also describes all kind of labeling algorithm early developed, and its drawbacks and advantages. However, it is hard to implement and manufacture by using embedding system. There are massed papers regarding the object recognition by using fuzzy, neural and image processing identification $[10,11,12,13,14,15]$.

$\mathrm{Hu}$ et al. [16] proposed a Chinese Chess Recognition algorithm based on projection histogram of polar coordinates image and Fast Fourier Transform. It adopts the rotation-invariant feature of chess characters. Experiments demonstrate that the algorithm can accurately recognize all chesses, and is robust to any rotation attacks.

In this paper, we develop an algorithm to calculate the features of the Chinese-chess image. It includes several stages: calculating starting point for synchronizing the comparison signals, creating a standard feature database, and identifying the input belonged image. The remainder of this paper is organized as follows: Section 2 gives a presentation of the relative work. Section 3 illustrates the algorithm of the Chinese-chess image recognition. Section 4 shows the experimental results. Section 5 presents the conclusions of this paper.

\section{Related Work}

Since Chinese-chess image identification has very high complicated operation, we define a feature of the Chinese-chess to reduce the complicity and simplify the processing. At the same time, a webcam is used to capture, zoom in, zoom out images so to result in deformation problems. Therefore, a normalization technique to unify those problems is needed. Since the DFT transform has a rotation invariant property, it is a suitable method for the Chinese chess recognition. The details will be described in the following.

\subsection{Feature Definition}

In this research, we measure the distance between the contour of the character and the center of the chess, which are used as features. Associated with our identification algorithm, it can exactly detect the Chinese-chess. In distance measurement step, we divide the contour of the chess image into 360 computation points corresponding to the 360 degree of chess. The 360 values are our features. Formula (1) is the equation used to calculate the distance feature.

$$
E(i)=\alpha \times \frac{\operatorname{Max}[\text { WContours }(i)]}{R},
$$

where $E(i)$ denotes distance feature between the word contours and center of the computation point $i, \alpha$ denotes the scale factor default value is $100, R$ express Radius, and WContours $(i)$ is the word contours of point $i$.

In feature calculation, we divide the chess into 360 computation parts, which means that each part of the chess object will generate a feature value. The feature value is generated by computing the distance between the character and the center of chess of the contours. We accumulate all the 360 data used as the feature of the chess, where data is expressed by curve for easier observation. The features will be used as the standard for the following input chess image comparison.

\subsection{Normalization}

For a Chinese chess, it is a circle contour inside where a Chinese character is. Therefore, it has the same measured radius regardless of the angle. Since the object image has zoom-out, zoom-in, and incline problems. A normalization step is necessary to make it on standard state for object image recognition. When used to compare, the condition and environment must be consistent to make results correct and meaningful. Therefore, our algorithm needs a normalization operation. In this research, we select a suitable radius value of the chess image used as a standard. Use a scale equation to adjust offset value. After scale equation adjustment, all of the objects with different shapes and sizes become consistent so to achieve normalization target. The formula of the scale method is listed in Equation (2). Fig. 1 shows the working theorem of the scale method. Fig. 1(a) is the selected standard image and Fig. 1(b) denotes the object image on incline state. Fig. 1(c) display the object zoom-out image. Finally, Fig. 1(d) shows the object zoom in image. In normalization step, we just use the scale method to adjust the object into standard; it solves those deformation problems, and makes chess recognition easy.

$$
k_{p}=\frac{R_{p}}{R_{S}}
$$

where $k_{p}$ is the scale factor, $R_{p}$ is the radius of the computation points corresponding to the 360 degrees of chess on input object, and $R_{S}$ is the radius of the computation points of chess in the standard database.

\subsection{Discrete Fourier Transform}

Because playing chess has a rotation image problem, therefore how to select a transform with rotation 


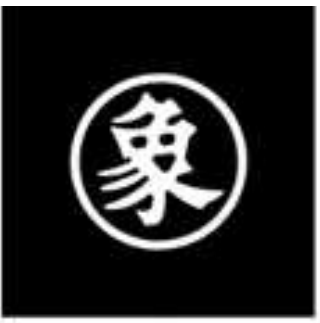

(a)

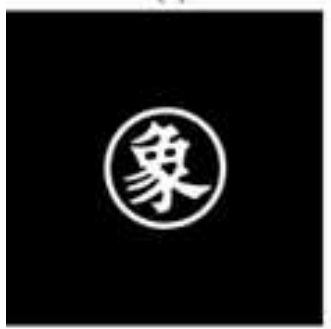

(c)

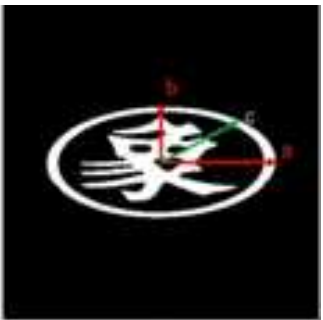

(b)

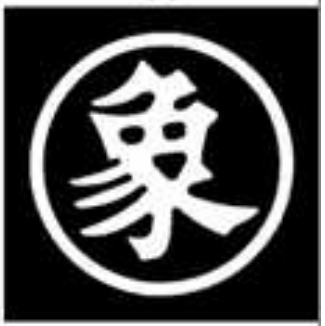

(d)
Fig. 1: The normalization schematic: (a) the selected standard image, (b) the object image on incline state, (c) the object zoomout image, (d) the object zoom in image.

invariance is important. Since the DFT transform has a rotation invariant property, it is a suitable method for the Chinese chess recognition. In this paper, we use one-dimension DFT to transfer the traits of the chess. The DFT formula is listed in the Equation (3)

$$
F_{U}=\frac{1}{N} \sum_{x=0}^{N-1} \exp \left[-2 \pi i \frac{x u}{N}\right] f_{x}
$$

where $F_{U}$ is the coefficient of the DFT, and $F_{x}$ is the value of the spatial domain.

In order to shows the DFT rotated invariant property, we make an example as shown in Fig. 2. Fig. 2(a) is the test image called object-1 and Fig. 2 (b) shows the distribution of the DFT corresponding to Fig. 2 (a). Likely, Fig. 2(c) is the test image called object-2 and Fig. 2(d) shows the distribution of the DFT corresponding to the Fig. 2(c). By careful analysis, in Fig. 2, we can see the DFT has exact rotation invariant property because DFT coefficients are always rotated according to its spatial domain shape.

\section{Recognition Algorithms}

In recognition algorithm, there are two phases to identify the Chinese-chess. One is the database creation phase; a set of standard database of the chess features for input object comparison need to be created. The other is the input chess comparison phase; the candidate chess object is pick up, and compare the object with data in database to distinguish which chess is.

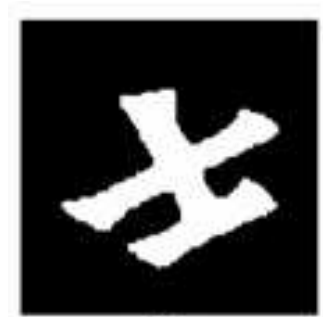

(a)

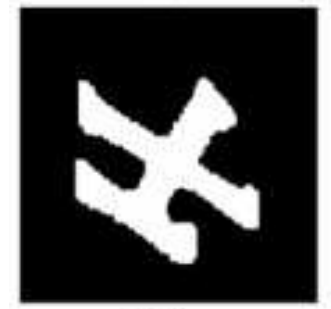

(c)

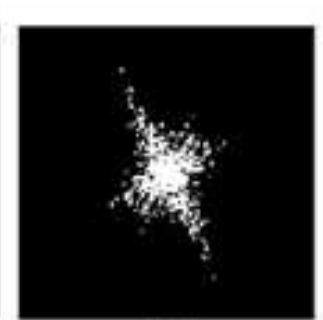

(b)

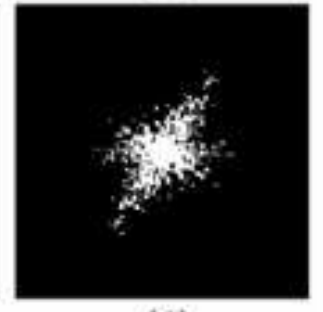

(d)
Fig. 2: The schematic of the DFT rotated invariance; (a) the object-1 image, (b) the distribution of DFT corresponding to (a), (c) the object-2 image, (d) the distribution of DFT corresponding to $(\mathrm{c})$

\subsection{Feature Comparison on Spatial Domain (FCSD)}

The overall identification process of the FCSD method is shown in Fig. 3. First, an image preprocessing stage is used to filter out the noise and improve image identification. The object extracting stage is used to get the target object. Because we use the distance between the contour of the character and the center of the chess as the feature, how to get the object center is an important step. Since the extracted chess object might not have a standard format, a scale method is used to make the object normalization and improve corrective identification. In feature calculation step, the chess object is divided into 360 computation parts and calculate each part distance from the center to object contour. Here we call the 360 distance features as energy wave of the chess, which are stored to the database for input image comparison. Besides, the input picture might be captured at a different orientation so that it causes different energy wave. Thus, we make the energy wave standardized by using maximum slop point of energy wave for consistent energy wave. In chess comparison phase, the steps such as image processing, object extraction, object center calculation, normalization and feature calculation and maximum slop points of the energy wave are the same as the database creation phase. Finally, a decision function is used to determine the input image is which chess by comparing the features between the input object and database. The details of the chess recognition are described in the following. 


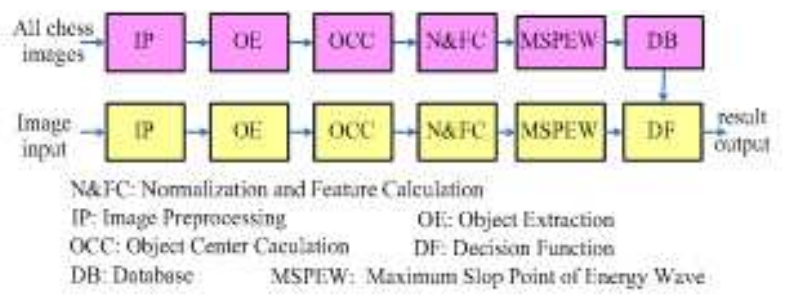

Fig. 3: The Chinese-chess recognition by FCFD algorithm.

\subsection{Image Preprocessing}

In order to filter out the noise and clean up the environment of the input image, the system needs an image preprocessing (IP) stage to hit the goal. The flow chart of the image pre-processing is shown in Fig. 4. At beginning, a RGB2YIQ color transform transfers the input color image from RGB to YIQ, which then is used to get the Y-plane for the following image processing. Since binary image is easier for object detection when compared with grayscale or color image. And then a suitable threshold used to convert the image in $\mathrm{Y}$ plane into binary format. For improving the identification effectively, a closing operation is used to filter out the noise. A labeling technique is used to mark all the objects. And an inappropriate area cancellation step is used to eliminate those interference blocks and leave useful objects. Successively, an area filled operation used to fill the objects. Finally, a candidate object selection stage is used to pre-select those candidate objects for the following object extraction.

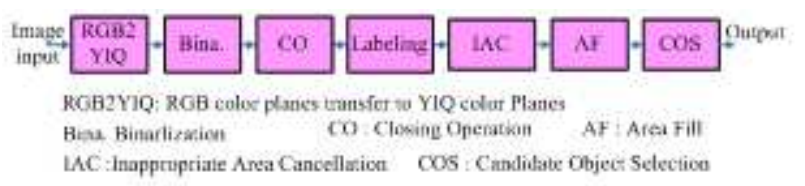

Fig. 4: The flow chart of the image pre-processing.

\subsubsection{Binarization}

In binarization step, we use the Y plane to convert the input image into binary image. We use the single threshold to convert the image in $\mathrm{Y}$ plan into binary image, and list it in equation (4).

$$
B_{Y}=\left\{\begin{array}{l}
1, Y \leq S_{\text {thY }} \\
0, \text { otherwise }
\end{array},\right.
$$

where $B_{Y}$ denotes the binary values which is corresponding to the point in the Y plane. $V_{Y}$ is the input image in $\mathrm{Y}$ plane, and $S_{t h Y}$ is the threshold used in binarization.

\subsubsection{Closing Operation}

Closing operation is a kind of Morphological operation; it can remove the notch and irregular small hole. It is similar to filter out the noise. In Chinese-chess recognition, for a lot of processing in binary image, a closing operation used to remove noise is appropriate. The closing operation formula is listed in equation (5). Fig. 5 shows the closing operation result. Fig. 5(a) is the binary image, and Fig. 5(b) shows the result image after closing operation corresponding to Fig. 5(a)

$$
A \cdot B=(A \oplus B) \odot B,
$$

where $A$ denotes an object to be processed and $B$ denotes a structure object. The notation - denotes closing operation, the notation $\oplus$ denotes dilation operation, and notation $\odot$ denotes erosion operation.

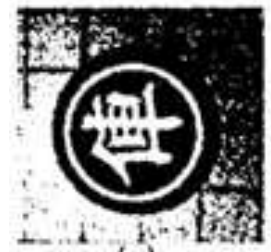

(a)

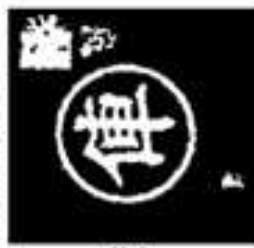

(b)
Fig. 5: The closing operation image: (a) the binary image, (b) the image after closing operation corresponding to (a).

\subsubsection{Labeling and Inappropriate Area Cancellation}

In object extraction, usually we use the labeling technique to count the number of objects and sum up pixels to which the object belongs. Besides, we use inappropriate area cancellation (IAC) method to eliminate those noises since objects have several noises which exist in the image. IN this research, we find that those objects sizes are too small and bigger ones are noise needed to be discarded. In IAC operation, we use formula (6) to filter out those inappropriate objects.

$$
B_{A}=\left\{\begin{array}{l}
1, S_{t h L}<A_{i}<S_{t h H}, i=1,2, \cdots, m \\
0, \text { otherwise }
\end{array}\right.
$$

where $B_{A}$ denotes the checking result of the IAC operation, parameters $S_{t h L}$ and $S_{t h H}$ are the threshold 
value for lower bound and upper bound of the IAC operation. The parameter $A_{i}$ is the pixel which belongs to one of the objects.

\subsubsection{Area Filling and Object Extraction}

To effectively extract objects, we first fill the labeling object. Because the object is filled, we can adopt the area size criterion easily to eliminate those not belonging to chess images. Fig. 6 shows the process of the area filling and candidate object image selection. Fig. 6(a) is the image after closing operation, and Fig. 6(b) shows the result image after area filling operation. Fig. 6(c) shows the selected candidate object image, and figure $6(d)$ is the extracted object image from mapping to Fig. 6(a).

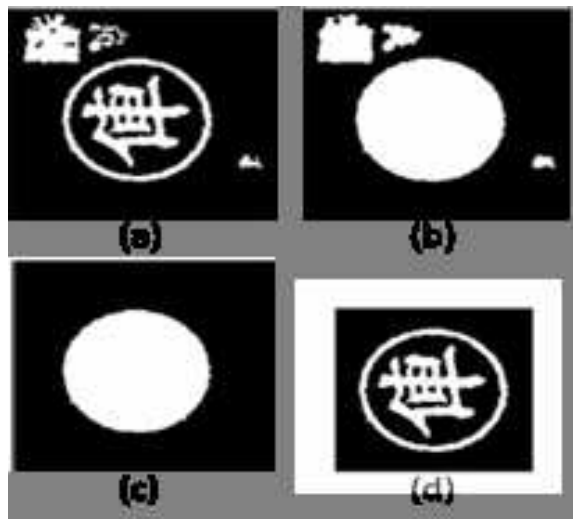

Fig. 6: The area filling and candidate object image selection: (a) the image after closing operation, (b) the image after area filling operation, (c) candidate object selection, (d) extracted object image.

\subsection{Object Center Calculation}

Owing to feature is calculating the distance from object contour to the center, thus, we need to get the object center first. Since the appearance of the Chinese-chess is circle, naturally, using the center of gravity $\left(x_{c}, y_{c}\right)$ as the center of the object is suitable. Eqs. (7)-(8) are the formulas used to compute the center of gravity $\left(x_{c}, y_{c}\right)$ of the chess.

$$
\begin{aligned}
& x_{c}=\frac{\sum_{i=1}^{b} x_{i}}{b}, \\
& y_{c}=\frac{\sum_{i=1}^{a} y_{i}}{a},
\end{aligned}
$$

where $x_{c}$ and $y_{c}$ are the coordinates of the center of gravity, respectively. The parameters $x_{i}$ and $y_{i}$ denotes the coordinates that belong to one of the object, respectively.

\subsection{Maximum Slop Point of Energy Wave and Feature Database}

In Session two, we have already described the Chinese-chess feature definition, calculation and normalization. The chess has several orientations when placed on the board, and, therefore, a maximum slop point of the energy wave (MSPEW) is used to synchronize the comparison signal.

\subsubsection{Maximum Slop Point of Energy Wave}

When playing Chinese-chess, it can be placed on the board in an arbitrary orientation. This will cause several different energy wave at different angular of the same chess. It might make the chess image detection fail. However, it is core work to find a method to standardize the energy wave and to make the energy wave rotation invariant. For solving this problem, we develop an average MSPEW method to achieve the goal. In MSPEW, we computer and record the slop of each point (one of 360 degree) and energy wave with the average slop. For example, assume the slops are 70, 80, 75, 80, 75, 70 corresponding to the angular point p1, p2, p3, p4, p5, p6 then the slop value of $\mathrm{p} 6$ will replace 70 with 75 . By using the average the slop, it has filter effect, can filter out the noise and obtain a corrective starting point. Since both input object and objects in database have the same starting point, the feature comparison can be correct and effectively executed.

In practical, we select the maximum positive slop point used as the comparison starting point; it is similar to the synchronization point of signal processing. Once the signals are synchronizing, then all the operation can be effective and meaningful. Meanwhile, the 360 energy waves on 360 measured angles on the same chess have the same value on rotate state. Therefore, the MSPEW method can standardize the starting energy wave of the chess, and calculating. The formulas to calculate starting point are listed by the equations (9)-(11).

$$
\begin{gathered}
m_{n}=\frac{y_{n}-y_{n-1}}{x_{n}-x_{n-1}}, \\
m_{p}=\frac{\sum_{k=n-5}^{n}}{6}, \\
p_{s}=\max \left(m_{p}, k=1,2,3, \cdots, 360\right),
\end{gathered}
$$

\subsubsection{Feature Database for FCSD method}

In Chinese-chess, there are 14 chesses including seven black color chess and seven red color chess. In our definition, chess has 360 energy features values. Thus, before starting the chess recognition process, we need to 
construct a database including 14 chess features. Fig. 7 shows the database content which is used for input object comparison. The database includes the fourteen chesses and their 360 feature curves.

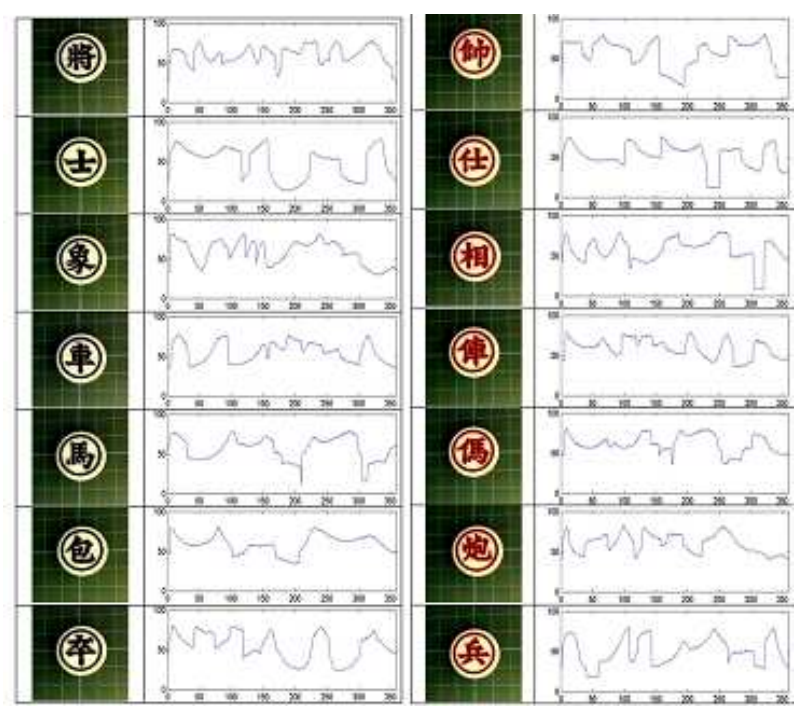

Fig. 7: The database of the Chinese-chess for FCSD method

\subsection{Decision Functions of FCSD Method}

For chess image identification, we need a decision function used to distinguish the input object image to which chess belongs. In FCSD method, the decision function is completed by two factors; one is the feature comparison, the other is the comparison error count. As for features comparison, we check and record the difference between input chess feature and standard feature of the database point by point. On comparison error count, we accumulate the number of difference value greater than threshold-1 (here $S_{t h D}$ is 10) through all 360 comparison points. Furthermore, we view the accumulated error number checking if the threshold-2 (here $S_{t h N}$ is 90) is over, and decide if the two objects are the same. The decision formulas are listed in the equations (12)-(14). The Equation (12) calculates the error of each measured angular comparison not matched with the database. If the difference is greater than or equal to $S_{t h D}$ we judge that they are different and $e_{P}=1$ is set. Then we count all the measure point from angular 0 to 360 and accumulate its difference value $e_{P}$, which is called $n_{e}$. Finally, Equation (14) judges if $n_{e}$ is greater than the threshold value $S_{t h N}$. Therefore, we can assure that the chess image is different from data in database, and checking next chess will continue on the database.

$$
\begin{gathered}
e_{p}=\left\{\begin{array}{l}
1,\left|v_{s}-v_{i}\right| \geq S_{t h D} \\
0, \text { otherwise }
\end{array}\right. \\
n_{e}=\sum_{p=1}^{360} e_{p}, \\
d_{f}=n_{e} \leq S_{t h N},
\end{gathered}
$$

where $e_{p}$ denotes the error bit of each computation point, parameters $v_{s}$, and $v_{i}$ are the feature value for data in database and input object of each computation point, respectively. The $S_{t h D}$ is the threshold used for judging each computation point if error happens. The $d_{f}$ is the error number of 360 computation points. The is the decision flag to indicate if the input object is the same as data in database. The $S_{t h N}$ is the threshold for $d_{f}$ flag decision.

\subsection{Feature Comparison on Frequency Domain (FCFD)}

At system beginning of the FCFD method, an image preprocessing (IP) step is used to filter out the noise. The object extracting (OE) is used to get the target object from image. A scale method is used to make the object normalized and improve correct identification. In features calculation step, we divide the chess object into 360 computation parts and calculate the distance of each part from the center to an object contour. Here we call the 360 distance as traits data of the chess, which are further passed to DFT transform to obtain features of the chess which are stored to the database for input image comparison. In chess detect and comparison phase, the steps such as IP, OE, CC, N\&FC and DFT DB are the same as the database creation phase. Finally, a decision function is used to compare the features between the input object and database, and determine to which chess the input image belongs. The details of the chess image recognition algorithm are described in the following.

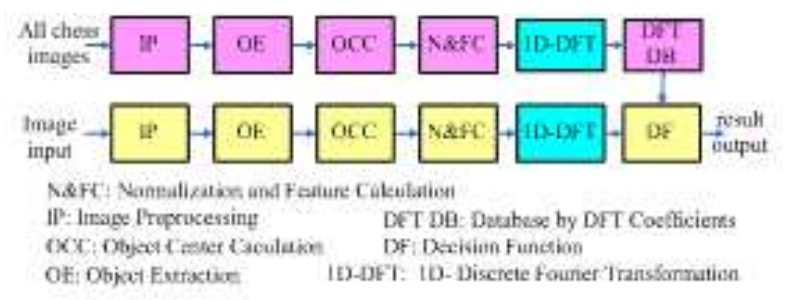

Fig. 8: The Chinese-chess recognition algorithm for FCFD method. 
Table 1: The first 10 data of the DFT coefficients on all the Chinese-chess.

\begin{tabular}{|c|c|c|c|c|c|c|c|c|c|c|}
\hline & F1 & $\mathrm{F} 2$ & F3 & F4 & F5 & F6 & F7 & F8 & F9 & F10 \\
\hline th & 9841 & 822 & 1307 & 223 & 877 & 825 & 802 & 449 & 618 & 200 \\
\hline 任 & 9166 & 423 & 501 & 158 & 660 & 415 & 676 & 1015 & 170 & 352 \\
\hline 相 & 9931 & 615 & 965 & 257 & 965 & 362 & 323 & 232 & 473 & 1016 \\
\hline 伊 & 10466 & 729 & 106 & 107 & 378 & 272 & 672 & 661 & 245 & 287 \\
\hline 值 & 10742 & 522 & 388 & 538 & 961 & 402 & 498 & 247 & 300 & 75 \\
\hline 垉 & 10745 & 650 & 552 & 472 & 504 & 175 & 301 & 264 & 541 & 379 \\
\hline 兵 & 8836 & 648 & 230 & 1243 & 304 & 227 & 617 & 737 & 636 & 821 \\
\hline 旃 & 10510 & 501 & 92 & 195 & 661 & 94 & 390 & 674 & 277 & 137 \\
\hline \pm & 8406 & 1398 & 568 & 915 & 1072 & 626 & 1052 & 179 & 871 & 278 \\
\hline 类 & 10162 & 518 & 1088 & 674 & 679 & 352 & 579 & 329 & 371 & 164 \\
\hline 7 & 9739 & 539 & 506 & 405 & 68 & 648 & 911 & 355 & 81 & 68 \\
\hline$B_{3}$ & 9909 & 185 & 593 & 1364 & 663 & 586 & 412 & 259 & 519 & 79 \\
\hline e & 10554 & 598 & 676 & 272 & 571 & 692 & 241 & 312 & 110 & 127 \\
\hline 4 & 9657 & 1183 & 137 & 348 & 793 & 1106 & 621 & 396 & 600 & 173 \\
\hline
\end{tabular}

\subsection{DFT coefficients of Trait Wave and Feature Database}

In Session two, we have already described the Chinese-chess feature calculation and normalization. The chess has several orientations when placed on the board. Therefore, a DFT transform is used to obtain the coefficients, which are rotation invariance of the trait data step and are needed to compare with the input object and database. In the Chinese chess, there are 14 kinds of chess characters including seven black color chess characters and seven red color chess characters. In FCFD, the features are DFT coefficients of the trait values of the chess characters. Therefore, before starting the chess recognition process, we need to construct a database including 14 chess features. Before the system identifies the input test chess, we use the 14 Chinese chess characters to simulate the features; each chess character is run on 20 different orientations. After we add up, the features database was completed. Table 1 shows the first 10 coefficients which is the trait data transfer by DFT.

\subsection{Decision Functions of the FCFD method}

In FCFD method, the DFT coefficient is denoted as F4 and $\mathrm{F9}$, are the factors we use as the features comparison for input chess recognition. During features comparison, we check and record the difference between input chess feature and standard feature of the database chess by chess. Finally, we check and select the minimum error chess used as its chess. In our method, the decision formulas are listed in the Equations (15)-(16). Equation (15) calculates the features error by accumulating the difference between the coefficients F4 and F9, which are the errors between an input test image and standard images in database. Finally, Equation (16) searches the minimum error the index $\mathrm{k}$ corresponding to the chess $\mathrm{k}$ is the same as the input test image.

$$
\begin{gathered}
e_{k}=2 \times\left|F 4_{i}-F 4_{S k}\right|+3 \times\left|F 9_{i}-F 9_{S k}\right|, \\
f_{k}=f\left(\text { mine }_{k}\right),
\end{gathered}
$$

where $F 4_{i}$ and $F 9_{i}$ are the features of the input test object. $F 4_{S k}$ and $F 9_{S k}$ denote the features of the chess $\mathrm{k}$ in database. Besides, $e_{k}$ denotes the feature error bit between input test object and chess $\mathrm{k}$ in the database. $f_{k}$ is the flag to indicate if the input test object is the same as chess $\mathrm{k}$ in the database.

\section{Empirical Results}

\subsection{Simulation Environmental}

In order to demonstrating the performance of the proposed scheme, all kind of Chinese chess images with size $640 \times 480$ were used in simulation., In simulation, we set parameters $s_{t h Y}=0.4, s_{t h D}=10$, and $s_{t h N}=90$, and we extract the chess image with size $120 \times 120$ for image identification. The details of the simulation results are described in the following.

\subsection{Simulation Results for FCSD Method}

Fig. 9 and Fig. 10 express that the test results all $100 \%$ identify the input chess image and its three types of rotated images for red chess and black chess, respectively. Since chess has 360 computation rotation points, due to paper limit, we randomly show three points of rotation results. After careful observation, we see that the energy waves are the same on starting point condition. Table 2 shows the testing results by using three types of rotating test point corresponding to figure 9 and figure 10. From the table, we see that the maximum error number is 85 , which is less than $S_{t h N}=90$, and therefore passes the detection. Fig. 11 shows the chess images and its features curve under 40 degree incline testing. Fig. 11(a) is the test images including incline and zoon-in testing. Fig. 11(b) expresses the original energy waves obtained by calculating on regular rules. Fig. 11(c) shows the energy wave after standardize starting point corresponding to the Fig. 11(b). It is obvious that the tests are all correct. Similarly, Fig. 12 shows the other testing image chess “ ", and it is similar to Fig. 11. With different chess, it also gets correct results. 


\subsection{Simulation Results for FCFD Method}

The simulation results of the FCFD method are shown in Fig. 13- Fig. 16. Fig. 13 displays one of test results of the chess image where it shows the rotation and zoon-in testing. From the testing results, we find the error is -0.0404 , which are less than threshold, so all are correctively identified. Fig. 14 shows one of test results of the chess image where it shows the rotation and zoon-out testing. Testing results reveal the error is 0.0658 , which are less than threshold, so all are correctively identified. Fig. 15 denotes one of test results of the chess image where it shows the $20 \%$ added noises. According to test results, it is shown that the chess has error is -0.034 less than the threshold. Therefore all are successful identified. Fig. 16 shows the chess images and its features under a 40 degree incline testing. According to test results, it is shown that the chess has error is 0.0441 less than the threshold. It is obvious that the tests are all correct. For each attack of chesses mentioned above, we simulated over 140 times of testing with a standard size, zoom-in, zoom-out, incline and added noises under all different orientations; and all of tests got correct identification. For the convenience to read, we list the simulation results in Table 3. Where the parameters of the Table 3 listed below: fea. $=2 * F 4+3 * F 9$, err.=fea. of input image -fea of database, and err\%=err. of the input image /fea of the database.

\subsection{Comparison with Other Methods}

Table 4 shows the all types of attacks comparison among the proposed method and others. On resisting the rotation attacks, all papers are all done. Hu [16] did not show the zoom-in or zoom-out test. As for the robustness to the pepper and salt attacks, Hu and AISYS [17] just did, but not describe. In incline attack, Hu did not display, and AISYS can resist 30 degrees attack, which is less than our method since we can pass 40 degrees incline attacks. According to Table 4 shown in, it is evident that our method is an effective and robust method for Chinese-chess recognition.

\section{Conclusions}

In this paper, the image zoom-in, zoom-out, rotation, incline image, and added noise are all used to simulation. In chess identification, we compare the features between the input image and image in database by two stages; one is to compare and record the 360 computing points; the other is to compare the accumulated error number of playing chess. From the experimental results, our algorithm by FCSD method and FCFD method are all achieve a $100 \%$ identification rate of the Chinese-chess. Meanwhile, it is show that our proposed scheme has two

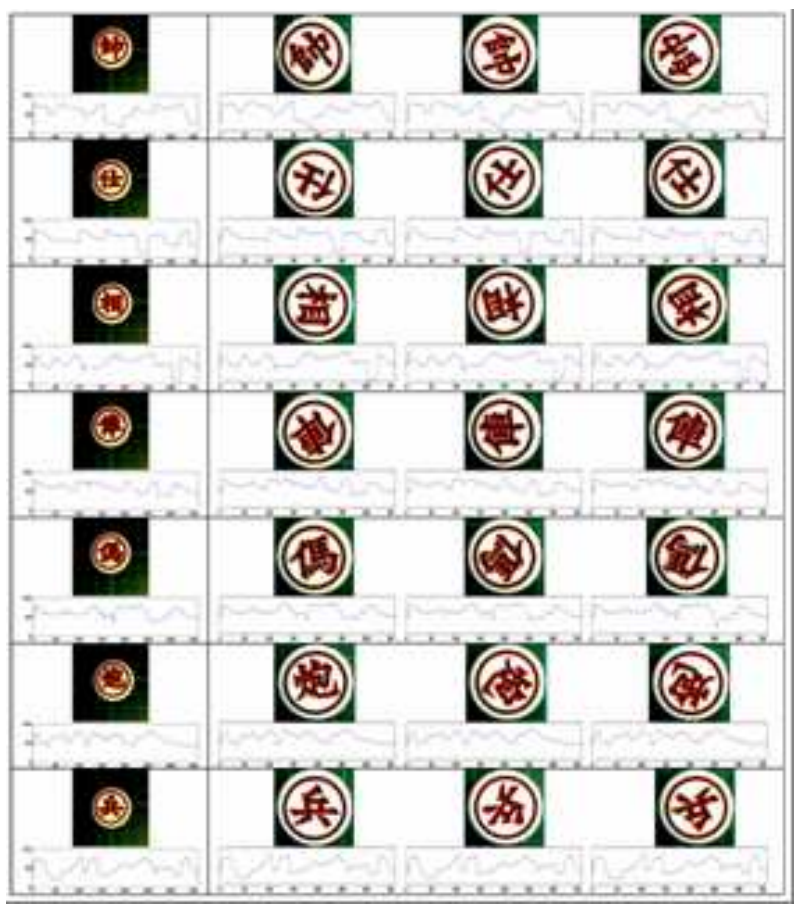

Fig. 9: The test results of the input chess image and its three types of rotated images for color red chess; (a) the original test image, (b) the test image on three kinds of rotation.

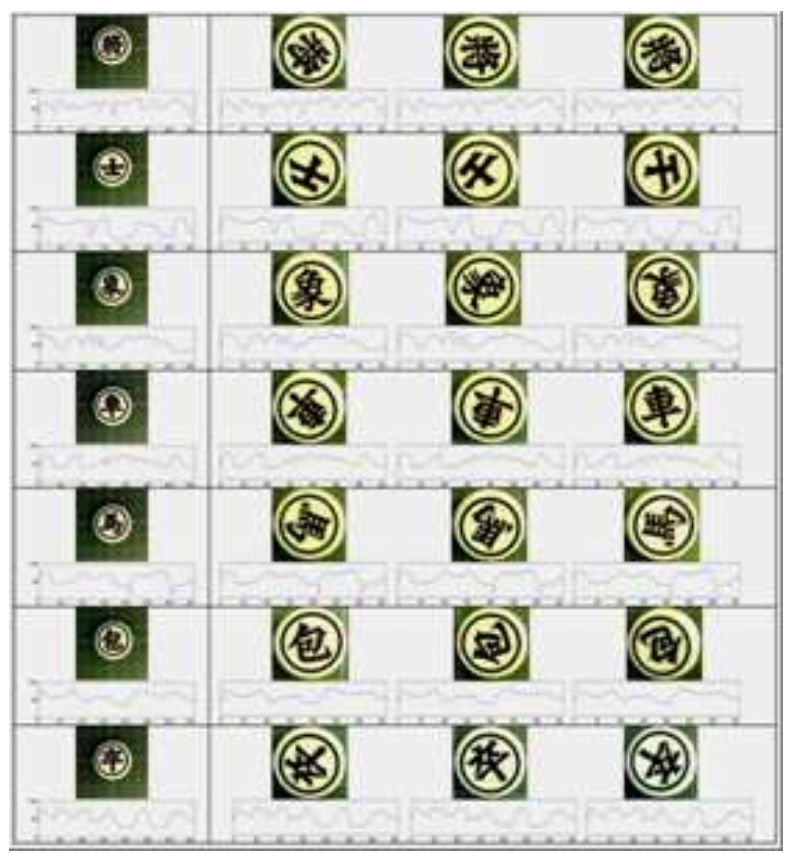

Fig. 10: The test results of the input chess image and its three types of rotated images for color black chess; (a) the original test image, (b) the test image on three types of rotation.

advantages when compared with other method: 1) our 
Table 3: The errors of the all kind of attacks on the proposed method.

\begin{tabular}{|c|c|c|c|c|c|c|c|c|c|c|c|c|c|}
\hline \multirow{2}{*}{ Item } & \multirow{2}{*}{$\begin{array}{c}\text { Standard } \\
\text { feature }\end{array}$} & \multicolumn{3}{|c|}{ Zoom in } & \multicolumn{3}{|c|}{ Zoom out } & \multicolumn{3}{|c|}{ Incline $40^{\circ}$} & \multicolumn{3}{|c|}{$20 \%$ noise } \\
\hline & & fea. & err. & err\% & fea. & err. & err\% & fea. & err. & err\% & fea. & err. & err $\%$ \\
\hline 的 & 2200 & 2095 & -105 & .9 .87 & 2246 & 46 & 2.09 & 2267 & 67 & 3.05 & 2146 & -54 & -2.45 \\
\hline 仕 & 910 & 933 & 23 & 2.16 & 959 & 49 & 5.38 & 865 & -45 & $-4,95$ & 1000 & 90 & 9.89 \\
\hline 相 & 1933 & 1890 & .43 & $-4,04$ & 1796 & -137 & .7 .09 & 1989 & 56 & 2.90 & 1949 & 16 & 0.83 \\
\hline 俥 & 980 & 919 & -61 & -5.73 & 928 & -52 & -5.31 & 1013 & 33 & 3.37 & 1068 & 88 & 8.98 \\
\hline 倩 & 2100 & 2130 & 30 & 2.82 & 2072 & -28 & -1.33 & 2209 & 109 & 5.19 & 2133 & 33 & 1.57 \\
\hline 炮 & 2567 & 2611 & 44 & 4.14 & 2614 & 47 & 1.83 & 2558 & 9 & -0.35 & 2641 & 74 & 2.88 \\
\hline 兵 & 4450 & 4545 & 95 & 8.93 & 4370 & -80 & -1.80 & 4663 & 213 & 4.79 & 4447 & -3 & -0.07 \\
\hline 特 & 1221 & 1315 & 94 & 8.83 & 1287 & 66 & 5.41 & 1260 & 39 & 3.19 & 1179 & .42 & $-3,44$ \\
\hline \pm & 4443 & 4497 & 54 & 5.08 & 4537 & 94 & 2.12 & 4502 & 59 & 1.33 & 4474 & 31 & 0.70 \\
\hline 象 & 2470 & 2461 & -9 & 0.85 & 2519 & 49 & 1.98 & 2406 & .64 & .2 .59 & 2476 & 6 & 0.24 \\
\hline 車 & 1153 & 1175 & 22 & 2.07 & 1043 & -110 & -9.54 & 1091 & -62 & .5 .38 & 1265 & 112 & 9.71 \\
\hline 典 & 4285 & 4257 & -28 & -2.63 & 4567 & 282 & 6.58 & 4579 & 294 & 6.86 & 4320 & 35 & 0.82 \\
\hline 包 & 962 & 863 & -99 & -9.30 & 934 & -28 & -2.91 & 1058 & 96 & 9.98 & 851 & -111 & -11.54 \\
\hline 卒 & 2496 & 2426 & -70 & -6.58 & 2432 & -64 & -256 & 2606 & 110 & 4.41 & 2524 & 28 & 1.12 \\
\hline
\end{tabular}

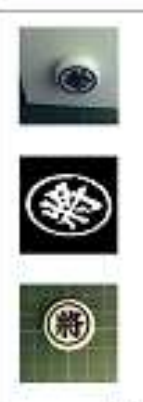

(a)

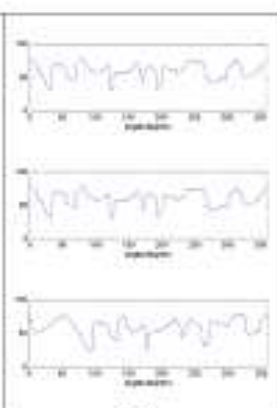

(b)

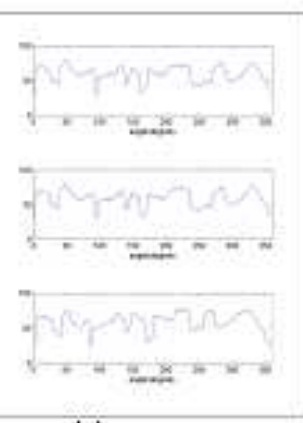

(c)
Fig. 11: The chess images and its features curve under 40 degrees incline test; (a) the test image, (b) the original energy wave, (c) the energy wave after standardizing starting point.

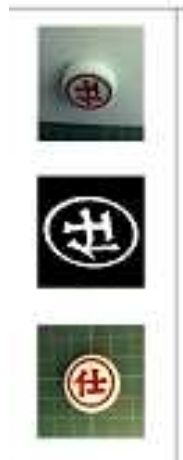

(a)



(b)



(c)
Fig. 12: The chess images and its features curve under 40 degrees incline test; (a) the test image, (b) the original energy wave, (c) the energy wave after standardizing starting point.
(24)

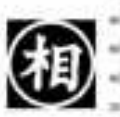

$+n+\infty+\infty+\infty$

Fig. 13: The chess images and its features in zoom in attack.

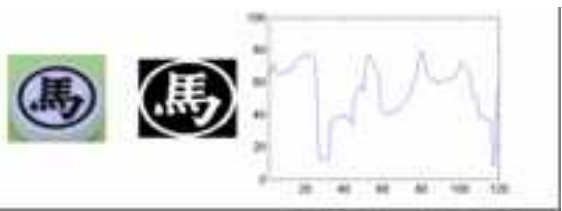

Fig. 14: The chess images and its features in the zoom-out attack.

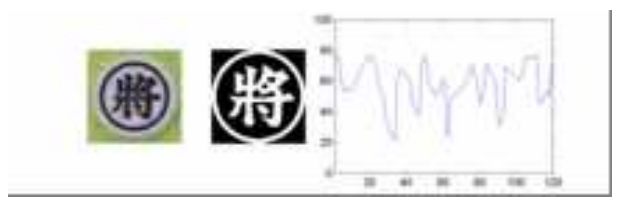

Fig. 15: The chess images and its features in $20 \%$ added noises attack.

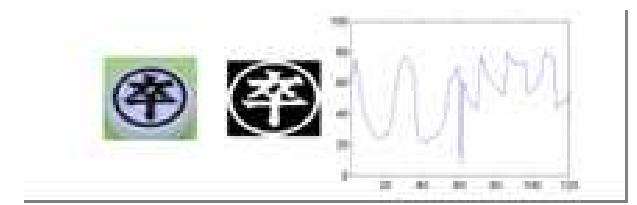

Fig. 16: The chess images and its features curve under 40 degrees incline attack. 
Table 2: The testing results by using three types of rotation corresponding to Fig. 9 and Fig. 10.

\begin{tabular}{c|ccc}
\hline \hline Item & $\begin{array}{c}\text { Test pattern-1 } \\
\text { error numbers }\end{array}$ & $\begin{array}{c}\text { Test pattern-2 } \\
\text { error numbers }\end{array}$ & $\begin{array}{c}\text { Test pattern-3 } \\
\text { error numbers }\end{array}$ \\
\hline \multirow{6}{*}{} & 31 & 56 & 27 \\
& 14 & 15 & 25 \\
& 58 & 44 & 20 \\
& 59 & 40 & 24 \\
& 33 & 24 & 58 \\
33 & 12 & 51 \\
& 29 & 19 & 37 \\
& 16 & 85 & 33 \\
& 33 & 41 & 25 \\
& 35 & 20 & 12 \\
& 38 & 36 & 85 \\
& 32 & 23 & 16 \\
& 7 & 48 & 8 \\
& 28 & 50 & 53 \\
\hline \hline
\end{tabular}

Table 4: All kind of attacks comparison among the proposed method and others.

\begin{tabular}{c|ccc}
\hline \hline Item & P. Hu [16] & $\begin{array}{c}\text { AISYS } \\
\text { Vision [17] }\end{array}$ & $\begin{array}{c}\text { Proposed } \\
\text { method }\end{array}$ \\
\hline $\begin{array}{c}\text { Robust to the } \\
\text { incline attack } \\
\begin{array}{c}\text { Resist the } \\
\text { rotation attacks }\end{array}\end{array}$ & No shows & Achieve 30 & Achieve $40^{\circ}$ \\
$\begin{array}{c}\text { Zoom in and } \\
\text { zoom out test }\end{array}$ & No shows & Yes & Yes \\
$\begin{array}{c}\text { Robust to the } \\
\text { pepper and salt } \\
\text { interference }\end{array}$ & No shows & No shows & Achieve 20\% \\
\hline \hline
\end{tabular}

method is robust against the 40 incline degrees attacks. It is better then other method only 30 degrees. 2) Our method can resist the $20 \%$ pepper and salt noise attacks.

\section{Acknowledgement}

This work was partly supported by the National Science Council, Taiwan (R.O.C.) under contract NSC 100-2221E-167-032.

\section{References}

[1] G. Tong, Y. Qu, T. Cheng, Human-Computer Interactive Gaming System-A Chinese Chess Robot, Intelligent Robots and Systems, 2009. IROS 2009. IEEE/RSJ International Conference, 984-987 (2009).
[2] C. Y. Lin, P. C. Jo, C. K. Tseng, Multi-Functional Intelligent Robot DOC-2, Humanoid Robots, 2006 6th IEEE-RAS International Conference, 530-535 (2006).

[3] J. Wang, L. Dong, X. Gao, C. Xu, F. Wang, C. Zhang, Research and Development of Super-Master-like Chess Playing Robot, Control and Decision Conference, 1332-1335 (2009).

[4] J. Wang, Y. H. Luo, D. N. Qiu, X. H. Xu, Adaptive Gbenetic Algorithm's Implement on Evaluation Function in Computer Chinese Chess, Proceedings of ISCIT2005, 12051209 (2005).

[5] Z. Chen, M. Liu, Z. Li, X. Lian, Key Technologies Analysis of Three Sides Chinese Chess Computer Game, IEEE conference, Second International Symposium on Intelligent Information Technology Application, 856-860 (2008).

[6] Y. F. Fan, X. J. Bai, R. Y. Liu, S. Xing, THE RESEARCH OF CHINESE CHESS BASED ON DATABASE WITH SELF LEARNING, Proceedings of the Ninth International Conference on Machine Learning and Cybernetics, 319-322 (2010).

[7] B. N. Chen, P. Liu, S. C. Hsu, T. S. Hsu, Integration of Chinese Chess Endgame Knowledge Bases, International Conference on Technologies and Applications of Artificial Intelligence, 450-455 (2010).

[8] D. Barik, M. Mondal, Object identification for computer vision using image segmentation, International Conference on Education Technology and Computer, 2, 170-172 (2010).

[9] L. He, Y. Chao, K. Suzuki, A Run-Based Two-Scan Labeling Algorithm, "IEEE Transactions on Image Processing, 17, 749-756 (2008).

[10] H. Zhu, J. Lei, X. Tian, A Pattern Recognition System Based on Computer Vision The method of Chinese chess recognition, IEEE International Conference on Granular Computing, 865-868 (2008).

[11] J. D. Foley, A. Van Dam, S. K. Feiner, J. F. Hughes, Fundamentals of Interactive Computer Graphics, second ed.Addison-Wesley, (1990).

[12] R. A. Hall, Illumination and Color in Computer Generated Imagery, Springer-Verlag, (1989).

[13] R. A. Hall, D. P. Greenberg, A testbed for realistic image synthesis, IEEE Computer Graphics and Applications, 3, 10-20 (1983).

[14] M. Zhai, S. Fu, Z. Jing, Homography estimation from planar contours in image sequence, Optical Enggineering, 49, 037202 (2010).

[15] T. C. Strand, Optical three-dimensional sensing for machine vision, Optical Enggineering, 24, 33-40 (1985).

[16] P. Hu, Y. Luo, C. Li, Chinese Chess Recognition based on Projection Histogram of Polar Coordinates Image and FFT, Pattern Recognition, 1-5 (2009).

[17] AISYS VISION, 2008, AISYS ChessReader, Retrieved May 3, 2010, from http://www.aisys.com.tw/web/product/. 


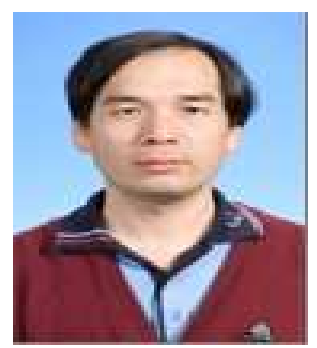

\section{Wen-Yuan Chen} was born in Taichung, Taiwan, in 1957. He received the B.S. and M. S. degrees in Electronic Engineering from National Taiwan University of Science and Technology in 1982 and 1984, respectively, and the Ph.D. degree in Electrical Engineering from National Cheng Kung University at Tainan Taiwan, in 2003. Since 2007, he has been a Professor in the Department of Electronic Engineering at National Chin-Yi University of Technology. His research interests include digital signal processing, image compression, pattern recognition and watermarking. 